\title{
The needs of family
}

members of patients

receiving radioactive iodine

By Margaret I. Fitch and Petrina N. McGrath

Funded by The Atomic Energy Board of Canada

\section{Abstract}

The current literature on radionuclide therapy is heavily based on health care professionals' perspectives and provides little insight regarding what patients or family members identify as their needs or concerns. Family members $(N=130)$ of patients undergoing treatment with radioactive iodine were selected from eight programs across Canada to participate in a survey to identify the needs they had when a close relative was undergoing treatment with radioactive iodine.

The results of the survey indicated family members experienced worries and concerns about the patient being at home after treatment, and there was variation in their knowledge about what precautions were needed. Family members provided evidence that many experienced psychosocial distress, but very few received adequate assistance for their difficulties.

Approximately 13,000 patients annually are treated in Canada with radioactive iodine (I131) for thyrotoxicosis or thyroid cancer (D. Dreidger, personal communication, March 4, 1998). When individuals are treated with radioactive iodine, they become a temporary source of ionizing radiation to other persons with whom they come in contact. Certain measures need to be taken to protect those who routinely interact with treated patients (Barrington et al., 1996; Barrington, Kettle, \& Thompson, 1996; Culver \& Cworkin, 1992). Radioactivity levels have been used to determine if a patient requires hospitalization and isolation.

In general, patients with thyroid cancer undergo surgery (i.e., partial or complete thyroidectomy) and hospitalization for their radionuclide therapy (Baker \& Feldman, 1993). Those with malignant disease receive higher doses of radionuclide therapy. Those with nonmalignant disease are treated with medication and receive their radionuclide therapy on an outpatient basis and implement specific protective procedures at home. A second or third radionuclide treatment may be required if the initial treatment dose fails to eradicate the thyroid tissue.

The inpatient group is usually subjected to protective isolation because of their higher dose therapy. Knowles (1993) indicated that there may be a difference in the experience of isolation between those for whom isolation is initiated for the protection of the patient versus those patients for whom isolation is imposed to protect others, as is the case of patients receiving I131. When isolated, the individual remains physically, socially, and emotionally concealed from the external environment. While not always necessary, it is common for patients who are in isolation to experience limited frequency and duration of contact with health care professionals and family members (Jenner, 1990). Very little research exists to help health care professionals understand the experience of isolation from either the patients' or family members' perspectives (Gaskill, Henderson, \& Fraser, 1997).

Family members of individuals who are diagnosed with an illness often experience fears and concerns. Treatment plans, procedures, medications, and meeting a range of health care professionals may all contribute to concerns and the impact experienced by the family member. The potential for being exposed to levels of radiation by their loved one may add another dimension to their concerns. Yet, there is little known about their possible fears.

Volunteers at the American Thyroid Foundation cited common concerns which lead patients with thyroid disease to call for help. The concerns included trying to understand therapeutic choices and concerns both after treatment and recovery (Wood, 1998). Individuals call the foundation because they want to talk with someone, they feel afraid, or they are too embarrassed to ask their physicians to repeat explanations. Family members call because they do not understand what the doctor said. The frequently reported fears specific to radioactive iodine were that radiation was dangerous to either oneself or the rest of the family, and that the treatment is a risk to the health and well-being of future children.

A number of studies have shown that adequate symptom control, available support services, relevant information, and meaningful communication with health care professionals are important to family members when a close relative is experiencing illness (Gray, Fitch, Davis, \& Phillips, 1997; McGrath \& Fitch, 2003; Reiker, Clark, \& Fogelberg, 1992; Wood, 1998). Although there are similarities across populations regarding these issues, the specific or unique needs of family members have not been documented for the interval when a close relative is receiving radioactive iodine therapy. Hence, this study was undertaken to identify the needs of family members when a close relative is undergoing treatment with radioactive iodine (I131).

\section{Methods}

Sample

To find family members, patients who had received radioactive iodine (I131) were selected initially for this cross-sectional descriptive study. The eligibility criteria for the patients included being: 1) older than 12 years of age, 2) diagnosed with malignant or non-malignant thyroid disease (no specific time interval since diagnosis), and 3) treated with any dose of I131. This group was selected because of the large number of patients treated with I131 and because the precautions taken during I131 therapy have been used as a model to guide the

Margaret I. Fitch, RN, PhD, is Head of Oncology Nursing and Supportive Care at Sunnybrook and Women's College Health Sciences Centre, Toronto, Ontario.

Petrina N. McGrath, RN, MN, is an Advanced Practice Nurse, Palliative Care, at Sunnybrook and Women's College Health Sciences Centre, Toronto, Ontario. 
approach to other radiopharmaceuticals. Each patient was asked to select a family member, defined as someone who was involved in their care and living at home with them during the course of their treatment, to participate by completing the study survey. There were no other predetermined eligibility criteria for the family members.

\section{Accrual}

A survey was used to gather data in this study. A total of 700 survey packages were distributed to physicians in eight clinics or sites across Canada where radioactive iodine therapy was delivered. Sites were selected purposively to reflect cross-Canada settings with major clinical programs delivering radioactive iodine therapy. The location sites included Newfoundland, Nova Scotia, Ontario (two sites), Quebec (two sites), Manitoba, and British Columbia. Ethics approval for the project was obtained from the respective ethics review boards of all sites. In each city, the physician was asked to participate. Upon receipt of the surveys, each of the eight physicians identified, from his or her caseload, 35 patients with a malignant diagnosis and 35 patients with a non-malignant disease who had received I131 treatment within the past two years. Although the I131 treatment was to have been received within the past two years, the actual diagnosis may have occurred a few months prior to the treatment event.

Survey packages were then mailed by the respective clinics to the selected patients. Each package contained a letter of explanation, a copy of the survey, and a pre-stamped return envelope. The patient gave the family member survey to the individual he or she selected. The family member completed the survey at home and returned it to the researcher's office. The researcher was not aware of the patient names or the family member names, and the physicians were not aware who had participated in the study. Given this arrangement requested by the ethics review boards, no follow-up reminders were sent.

\section{Survey instrument}

The survey instrument used in this work was developed for the purposes of this study. A patient survey was crafted following indepth interviews with 21 patients who had undergone treatment with I131. Results from the patient survey are reported in a separate publication (McGrath \& Fitch, 2003). The family member survey was crafted to match the content areas of the patient survey. The format of the survey was based on a survey format used successfully with prostate cancer patients (Gray, Klotz, et al., 1997) and ovarian cancer patients (Fitch, Gray, DePetrillo, Franssen, \& Howell, 1999). The survey was reviewed for content or face validity, readability, and clarity by individuals with survey development expertise and expertise in radioactive iodine treatment.

The survey was designed to elicit family members' perspectives regarding the following topic areas: experiences during diagnosis and treatment, including problems experienced and assistance received for problems; access to, importance of, satisfaction with information and communication with health care professionals; impact on the family member; and support. The survey items were precoded with response options generated from the ideas embodied in the indepth interviews. The majority of the items presented a list of response options and the respondents marked those which applied to them. Likert-type scales were used to assess the importance of information and the impact of the diagnosis and treatment on lifestyle. Opportunities to write additional responses were offered throughout the document. This paper reports the data regarding family member perspectives on access to information, experiences during treatment, precautions, impact on the family member, and support 


\section{Data analysis}

Data analysis for the survey was descriptive. Data from family members whose relatives were treated with I131 as inpatients as opposed to outpatients were handled and analyzed separately, given that the treatment protocols differ for the two patient groups. Item means, medians, and frequencies were calculated. The responses to the open-ended items were reviewed and subjected to a standard content analysis. Examples of the open-ended comments are reported to illustrate specific points.

\section{Results}

\section{Demographic characteristics}

A total of 130 family members returned the survey (response rate of 19\%). Sixty-eight had had a close relative treated as an inpatient and 62 had a close relative treated as an outpatient. Table One presents the demographic information from the family members in each group.

Overall, the respondents had an average age of 46.3 years (range 20 to 82). Half of the respondents were female and $83 \%$ were Caucasian. Almost two-thirds were partners (spouses) to the patients who received treatment. The majority had completed high school and half had also completed community college or university. Slightly more than half were working and $14 \%$ were retired. Approximately one-third (34\%) had an income between $\$ 30,000$ and $\$ 59,999$. The respondents were well-distributed across communities ranging from populations of less than 5,000 (22\%) and 5,000-49,000 (28\%) to $50,000-249,000$ (28\%). Responses were received from each of the six provinces where surveys were distributed.

Of the family members who had a patient treated as an inpatient, $66 \%$ of those patients had malignant disease. Fifty-three per cent of the inpatients had been diagnosed within the last year, while $32 \%$ had received their diagnosis between two and three years ago. Of the family members who had had a patient treated as an outpatient, 86\% had been diagnosed with non-malignant disease. Half of the outpatient group had received the diagnosis in the past year, while $29 \%$ learned of their diagnosis two to three years prior to the survey.
Family members for the inpatient group reported that, in addition to the radioactive iodine treatment, $84 \%$ of the patients had undergone a total thyroidectomy. Fifty-nine per cent of these patients underwent a single radioactive iodine treatment. The family members of the outpatient group indicated $50 \%$ of the patients had received medication in addition to the radioactive iodine therapy. In this group of patients, $82 \%$ experienced a single radioactive iodine treatment.

\section{Access to information}

In the six weeks following the patient's diagnosis of a thyroid condition, approximately half of the inpatient family members (54\%) and slightly less than half of the outpatient family members (44\%) felt they had someone with whom to talk about the diagnosis. Somewhat fewer felt they had someone with whom to talk about the treatment $(49 \%$ and $36 \%)$. The inpatient family members reported they could talk most frequently with general surgeons (40\%) and family physicians (34\%). Twenty-one per cent indicated there was no one with whom they could talk. The outpatient family members reported they could talk with family doctors (42\%) and endocrinologists $(24 \%)$. Twenty-seven per cent indicated they did not have anyone with whom they could talk.

Approximately two-thirds of the inpatient family members (62\%) and half of the outpatient family members (53\%) felt they had received a clear explanation of how radioactive iodine treatment works. Approximately $20 \%$ in both groups reported difficulty understanding treatment options and talking with health care professionals. The following comments reflect some of the difficulties:

For such a critical illness/surgery, medical personnel should take more time and make more effort to ensure the patient and their spouse/partner are better informed about the illness, the treatment, the after-effects, etc. We felt very "under-informed". Medical personnel may be always busy, but they need to take more time with patients who have critical surgery such as thyroid cancer.

Table Two: Information family members reported as important and their satisfaction with the information received

\begin{tabular}{|c|c|c|c|c|}
\hline \multirow[b]{2}{*}{ Topic } & \multicolumn{2}{|c|}{ Family members of inpatients $(n=68)$} & \multicolumn{2}{|c|}{ Family members of outpatients $(n=62)$} \\
\hline & $\begin{array}{l}\text { Percentage } \\
\text { adicating the topic } \\
\text { was important* }\end{array}$ & $\begin{array}{c}\text { Percentage } \\
\text { satisfied with the } \\
\text { information received } * *\end{array}$ & $\begin{array}{c}\text { Percentage } \\
\text { indicating the topic } \\
\text { was important* }\end{array}$ & $\begin{array}{c}\text { Percentage } \\
\text { satisfied with the } \\
\text { information received } * *\end{array}$ \\
\hline Family member's medical condition & 91.2 & 54.4 & 91.9 & 56.5 \\
\hline Planning for tests and procedures & 77.9 & 52.9 & 83.9 & 56.4 \\
\hline Treatment choices available & 80.9 & 50.0 & 90.3 & 61.3 \\
\hline Possible side effects of treatment & 83.8 & 50.0 & 87.1 & 51.6 \\
\hline Possible emotional reactions & 73.5 & 27.9 & 64.5 & 41.9 \\
\hline What to do to relieve physical discomfort & 60.3 & 29.4 & 79.0 & 43.5 \\
\hline Diet and nutrition & 67.6 & 29.4 & 62.9 & 41.9 \\
\hline Counselling services available & 44.1 & 17.6 & 30.6 & 33.9 \\
\hline $\begin{array}{l}\text { How to arrange to speak with another } \\
\text { person with the same thyroid condition }\end{array}$ & 42.6 & 16.2 & 35.5 & 29.0 \\
\hline Preparing for hospitalization & 69.1 & 48.5 & 32.3 & 37.1 \\
\hline Preparing your home after treatment & 64.7 & 38.2 & 53.2 & 43.5 \\
\hline
\end{tabular}


Not enough information received about risks and side effects of radioactive therapy.

I believe that any patient should be encouraged to invite and have present another family member in discussion of diagnosis and treatment.

The majority of family members in both the inpatient $(>77 \%)$ and outpatient $(>83 \%)$ groups rated information about the patient's medical condition, tests and procedures, treatment choices, and side effects of treatments as important for them to know as a family member (see Table Two). Two-thirds indicated topics such as emotional effects, diet and nutrition, preparing for hospitalization, and preparing one's home for the time after treatment were also important for family members to know. Within both family member groups, no more than about half of the respondents indicated they felt satisfied with the information they had received, with the exception of the information on treatment options $(61 \%)$, the patient's medical condition $(57 \%)$, and tests and procedures $(57 \%)$. The following comments reflect the difficulties family members felt:

People should be made aware of what radioactive iodine treatment is. My spouse was sent home from outpatient treatment with very little information on what he should and shouldn't do. I have to admit I was very nervous being around him.

I feel my husband's health was taken rather lightly, just another illness. We were very misinformed. No support was offered and there was an extreme lack of explanation. Luckily, my husband had the initiative to get information off the internet.

\section{Experiences during treatment}

The family members who had a patient treated as an inpatient reported that $96 \%$ of the patients were isolated. About a third $(34 \%)$ were isolated for one to two days and a half $(53 \%)$ were isolated for three to four days. During the isolation, $63 \%$ of the patients were not allowed to have visitors and $25 \%$ were able to have limited visiting.

Forty-four per cent of the family members reported difficulty surrounding the patient's isolation as an inpatient. The difficulty arose from not being able to be close to the patient or feeling separated. From the perspective of the family member, the aspects of the experience that made it easier for them as a family member during the isolation interval

Table Three: Precautions patients took at home following radioactive iodine treatment as reported by family members

\begin{tabular}{|l|c|c|}
\hline & \multicolumn{2}{|c|}{ Percentage of patients } \\
\hline Precaution & $\begin{array}{c}\text { Received } \\
\text { treatment as } \\
\text { an inpatient } \\
\text { (n=68) }\end{array}$ & $\begin{array}{c}\text { Received } \\
\text { treatment as } \\
\text { an outpatient } \\
\text { (n=62) }\end{array}$ \\
\hline Flushed toilet twice & 75.0 & 69.4 \\
Drank lots of fluids & 73.5 & 54.8 \\
Avoided contact with & 72.1 & 62.9 \\
children/pregnant women & 66.2 & 75.8 \\
Washed hands thoroughly & 61.8 & 48.4 \\
Slept alone & 60.3 & 62.9 \\
Avoided kissing/hugging & 58.8 & 46.8 \\
Kept away from others & 47.1 & 32.3 \\
Washed clothing/linen separately & 42.6 & 51.6 \\
Used own utensils or plastic utensils & 42.6 & 30.6 \\
Stayed alone or apart from family & 41.2 & 30.6 \\
Showered frequently & & \\
\hline
\end{tabular}

were: knowing that the isolation was only for a few days (69\%), being able to phone the patient (68\%), being able to have a short visit with the patient $(25 \%)$, and the interaction with the nurse $(21 \%)$.

\section{Precautions}

Family members were asked to report on the precautions which were put into place upon the patients' return home. Table Three presents the list of precautions reported by the family members. The most frequently identified precautions for patients following treatment as an inpatient included flushing the toilet twice (75\%), drinking lots of fluids (74\%), and avoiding contact with children or pregnant women $(72 \%)$. For the patients treated as an outpatient, the family members identified washing hands thoroughly (76\%) and flushing the toilet twice $(69 \%)$ most frequently.

The family members indicated they experienced difficulty with some aspects of the precautions. For family members of inpatients, the family members had difficulty with the patient being off thyroid medication before treatment (49\%), with the patient being in hospital $(47 \%)$, and not being able to get too close to the patient $(44 \%)$. The family members of outpatients found it difficult not to get too close to the patient $(21 \%)$, the patient being off medications $(19 \%)$, and the person being isolated $(15 \%)$. When patients were off their thyroid medication, family members had to cope with patients' mood swings, irritability, fatigue, and depression. In a few instances, the patients were instructed to remain in a room by themselves for a time at home.

Family members experienced concerns about the patient being at home after the radioactive iodine therapy. The largest proportion of family members in both the inpatient and the outpatient groups identified a concern about whether the treatment worked $(82 \%$ and $76 \%$ ). The next highest proportion identified a concern about how to know whether the radiation was gone from the home environment (54\% and $45 \%)$. Some of the confusion family members experienced is indicated in the following comments:

When A. had radioactive iodine therapy, he wasn't admitted to hospital. Some people have to be isolated. I was kind of concerned about that and it's nice to know the difference if you do or don't.

...my partner had two outpatient radioactive treatments and $\mathrm{NO}$ precautions were told to us.

\section{Impact of illness and treatment on family member}

The family members reported experiencing a range of difficulties themselves during the interval of the radioactive iodine therapy. Anxiety was identified by more than half of the family members in the inpatient (62\%) and the outpatient $(52 \%)$ groups. Both groups identified the same types of issues including feeling apart from the patient, fear, and feeling down or depressed. When asked whether or not they had received adequate assistance for the problems that they had experienced, there was considerable variation in the number of individuals who felt they had received adequate help.

\section{Support}

Forty-four per cent of the family members in the inpatient group reported that they had felt the need to talk about issues related to the illness and the treatment. Of those who felt the need, $67 \%$ indicated they had someone with whom they could talk. Twenty-one per cent indicated they talked with another family member, while $13 \%$ spoke with their family physician. Two (3\%) had seen a professional counsellor about issues concerning the patient's thyroid condition. Of the group who had not seen a counsellor, $22 \%$ indicated they would have liked to have seen someone. No one in this group had attended an educational or group session about thyroid disease. Twentynine per cent indicated they would have liked to have had that opportunity. 
As for the family members in the outpatient group, 57\% indicated they had felt the need to talk with someone about the patient's thyroid disease. Of those who had felt the need to talk, $86 \%$ indicated they had someone with whom they could talk. Fifteen per cent indicated they could talk with other family members and $15 \%$ indicated they talked with their family physician. Three $(5 \%)$ family members had been to see a professional counsellor regarding issues about the thyroid disease. Of those who had not seen a counsellor, $15 \%$ indicated they would have liked to have done so. Three (5\%) individuals had also participated in an educational or group session about thyroid disease. Of those who had not attended such a session, $16 \%$ indicated they would have liked to have done so.

\section{Discussion}

This study was conducted to gather perspectives from family members of patients who had received I131. Family members are integral to the care of the patient, particularly in the home environment.

There are several limitations in this study. One limitation is the small convenience sample. The distribution of the surveys occurred according to the original plan and surveys were returned from all locations where they were distributed. Although the response rate actually exceeds those of general mailings without reminder notices, generalizability of the findings may be somewhat limited.

Another limitation, as is the case with all mailed surveys, is that those who returned the completed forms are likely those individuals with an interest in the topic or individuals who have the ability to read and articulate responses to a document such as the survey instrument. Thus, the findings may under-represent the views of individuals who are less educated and individuals who lack facility with English or French. This sample is also not reflective of the wide cultural and ethnic community within Canada.

Finally, the data are subject to recall bias because of the retrospective nature of the design. Although the majority of family members were recalling experiences that occurred within the last year, some were reflecting upon events of two-and-a-half to three years ago.

Despite the limitations, this is the first Canada-wide survey regarding the perspectives of family members of individuals who have received radioactive iodine treatment. Responses were received from all provinces in which distribution occurred, and included a good cross-section of community sizes. The results provide interesting insights and offer implications for future investigations. The data provide evidence that the disease and treatment of a close relative have an impact on the family member as well as on the patient.

The family members did not express the same level of satisfaction with access to information as did the patients in the companion study (McGrath \& Fitch, 2003). This likely relates to their access to and interaction with health care professionals. They may have had to depend upon the patient to share and interpret information for them. Overall, they perceived information as important, yet they were less than satisfied with what they received across all topic areas. Similar findings have been reported in other work (Dow, Ferral, \& Anello, 1997). Clearly, there are implications in these observations for health care providers about communicating with family members and about providing written information regarding a range of topics related to radioactive iodine therapy. Many of the topics family members wanted to know more about were the same as those identified by patients (McGrath \& Fitch). The participation of nurses, nutritionists, and social workers on the teams providing care for patients receiving I131 and their respective family members could help with this information gap.

Many respondents in both the inpatient and the outpatient group indicated they felt informed about the disease and side effects and felt they had someone with whom they could talk about these matters. However, there were groups of respondents who did not have the explanations they felt they needed and did not have individuals with whom to talk. The challenge for health care providers is to be able to identify the individuals who need more information and find approaches to meet their needs.

At first glance, it is somewhat surprising that precautions were not reported as used by $100 \%$ of the respondents. However, the proportion of respondents citing the precautions used could reflect a number of factors: what was observed at the time, what the patient was told to do, what the family member remembers the patient doing, the policies in use at a particular institution, and the environmental reality (i.e., physical set-up in hospital). Also, there are variations in practices between inpatient and outpatient protocols. This variation possibly relates to the different dosage levels for patients with malignant disease and the amount of thyroid tissue the patient possesses. Nevertheless, there is a need for evidence-based guidelines regarding the precautions to be used in the home setting and sound educational strategies for sharing this information with patients and family members.

Of interest, many of the concerns family members expressed about the radioactive iodine therapy were the same as those expressed by patients (Kilpatrick, Kirstjanson, Tataryn, \& Fraser, 1998; McGrath \& Fitch, 2003) and family members in previous work (Wood, 1998). Primarily, they worried about whether the radiation was gone from the home and whether the treatment worked. Both issues are difficult aspects for family members to assess or judge easily. Family members also experienced difficulties around the patient being off medication, the patient being away in hospital, and issues of not being able to get "too close" to the patient. In all likelihood, interventions could be developed to help both patients and family members with these concerns.

Family members reported experiencing their own difficulties during the radioactive iodine treatment. Many of the reported difficulties are indicative of psychosocial distress (i.e., anxiety, fear, feeling down) and have been reported by family members of cancer patients in previous work (Hilton, 1996; Kristjanson \& Ashcroft, 1994; Northouse, Cracchiolo-Caraway, \& Appel, 1991; Walker, 1997). It is concerning that so few family members felt they received adequate help with these issues. It is unclear from the survey data whether family members were in contact with health care professionals regularly, whether the issues were identified by health care professionals, whether interventions were implemented, or whether interventions were not effective. Further work is needed to pinpoint the specific gaps in the care delivery process for family members.

Fairly large proportions of family members expressed the need to talk with someone about issues related to the disease and treatment. Although a sizable number of those individuals had someone with whom they could talk, a proportion did not have anyone. Very few family members had the opportunity to see a professional counsellor, attend a support or educational group, or access the Thyroid Foundation of Canada or the Canadian Cancer Society. These observations have implications for family education and providing information about community services.

\section{Summary}

This Canada-wide survey provided perspectives from family members of patients who received radioactive iodine therapy. Data indicate there is variation in family member perceptions about how precautions are to be implemented. Respondents expressed a desire for more information regarding many aspects of the treatment experience. Additionally, there are implications for support of family members in terms of dealing with psychosocial issues which emerge when an individual is living in the shadow of thyroid disease. 


\section{References}

Baker, K.H., \& Feldman, J.E. (1993). Thyroid cancer: A review. Oncology Nursing Forum, 20(1), 95-104.

Barrington, S.F., Kettle, A.G., O’Doherty, M.J., Wells, C.P., Somer, E.J.R., \& Coakley, A.J. (1996). Radiation dose rates for patients receiving iodine-131 therapy for carcinoma of the thyroid. European Journal of Nuclear Medicine, 23(2), 23-30.

Barrington, S.F., Kettle, A.G., \& Thomson, W.H., (1996). RPC Guidelines on radiation protection following radioactive iodine therapy for thyroidtoxicoisis: Are they appropriate? Nuclear Medicine Communications, 17, 276.

Culver, C.M., \& Cworkin, H.J. (1992). Radiation safety considerations for post iodine-131 thyroid cancer therapy. Journal of Nuclear Medicine, 33(7), 1402-1405.

Dow, K.H., Ferral, B.R., \& Anello, C. (1997). Quality of life changes in patients with thyroid cancer after withdrawal of thyroid hormone therapy. Thyroid, 7(4), 613-9.

Fitch, M.I., Gray, R.E., DePetrillo, A.D., Franssen, E., \& Howell, D (1999). Canadian women's perspectives on ovarian cancer. Cancer Prevention and Control, 13(1), 52-60.

Gaskill, D., Henderson, A., \& Fraser, M. (1997). Exploring the everyday world of the patient in isolation. Oncology Nursing Forum, 24(40), 695-700.

Gray, R.E., Fitch, M.I., Davis, C., \& Phillips, C. (1997). A qualitative study of breast cancer self-help groups. Psychooncology, 6, 279289.

Gray, R.E., Klotz, L.H., Iscoe, N.A., Fitch, M.I., Franssen, E., Johnson, B.J., \& Labrecque, M. (1997). Results of a survey of Canadian men with prostate cancer. The Canadian Journal of Urology, 4(2), 250-258.
Hilton, B.A. (1996). Getting back to normal: The family experience during early stage breast cancer. Oncology Nursing Forum, 23, 605-614.

Jenner, E. (1990). Aspects of isolation care. Nursing, 4(20), 17-22.

Kilpatrick, M.G., Kirstjanson, L.T., Tataryn, D.J., \& Fraser, V.H. (1998). Information needs of husbands of women with breast cancer. Oncology Nursing Forum, 25(9), 605-614.

Knowles, H. (1993). The experience of infectious patients in isolation. Nursing Times, 89(30), 3-56.

Kristjanson, L.J., \& Ashcroft, T. (1994). The family cancer journey: A literature review. Cancer Nursing, 17, 1-17.

McGrath, P., \& Fitch, M. (2003). Patient perspectives on the impact of receiving radioactive iodine: Implications for practice. Canadian Oncology Nursing Journal, 13(3), 152-156.

McGrath, P., et Fitch, M. (2003). "L'impact de l'administration d'iode radioactif depuis la perspective des patients : les répercussions pour la pratique". Revue canadienne de soins infirmiers en oncologie, 13(3), 157-163.

Northouse, L.L., Cracchiolo-Caraway, A., \& Appel, C.P. (1991). Psychological consequences of breast cancer on the partner and family. Seminars in Oncology Nursing, 7, 216-223.

Reiker, P., Clark, E., \& Fogelberg, P. (1992). Perceptions of quality of life and quality of care for patients with cancer. Oncology Nursing Forum, 19, 433-440.

Walker, B.L. (1997). Adjustment of husbands and wives to breast cancer. Cancer Practice, 5(2), 92-8.

Wood, L.C. (1998). Support groups for patients with Grave's disease and other thyroid conditions. Endocrinology Metabolic Clinics of North America, 27(1), 101-107. 